La construction du capital-marque des e-marques

Géraldine Michel $^{*}$ et Jean-François Vergne ${ }^{* *}$

Géraldine MICHEL, IAE Paris, Sorbonne Business School

Jean-François VERGNE, IUT Cergy Pontoise

* Maître de Conférences à l'IAE de Paris, Centre de Recherche du GREGOR.

*** Maître de Conférences à l’IUT de Cergy-Pontoise. 


\section{La construction du capital-marque des e-marques}

Résumé : L’objectif de cet article est d’étudier les spécificités des e-marques, marques créées uniquement sur Internet, et plus particulièrement de mieux comprendre la construction de leur capital-marque. Dans cette optique, les différents types de e-marques développés sur Internet sont présentés et les résultats d’une étude qualitative menée auprès de seize consommateurs sont exposés. Cette étude exploratoire montre que les e-marques connaissent des difficultés pour développer une image de marque précise, et révèle qu’elles développent un capitalmarque fondé sur des dimensions différentes selon le type de site Internet qui leur est associé. Des implications managériales sont alors développées.

Mots clés : E-marque, Internet, Capital-marque, Bénéfice fonctionnel, Bénéfice expérientiel

\section{Building the brand equity of an e-brand}

Abstract : The purpose of this paper is to study the specific characteristics of the e-brands (these brands which are only created on the Internet) and to better understand the brand-equity building of an e-brand. In this perspective, the different types of e-brands are exposed and a qualitative study, conducted with sixteen consumers, is presented. The results of this exploratory study reveal the e-brand difficulties to develop a distinct image. The study shows, also, that the brand equity is based on different dimensions according to the type of web site. This article formulates some recommandations for managers.

Key-words : E-brand, Brand-equity, Internet, Functional benefit, Experiential benefit 


\section{INTRODUCTION}

Depuis des années, maintes recherches s’intéressent au management de la marque. L’ensemble de ces études a permis de définir plusieurs concepts fondamentaux pour la gestion de la marque : l'identité de marque, l'image de marque, le noyau de la marque, le capitalmarque, le territoire de marque, etc. Peu de recherches ont jusqu’ici été consacrées aux problématiques de la marque sur Internet. Pourtant, les marques se trouvent aujourd'hui confrontées à de nouveaux défis face au développement d’Internet, leur rôle pouvant être fragilisé ou bien renforcé par l’arrivée de ce nouveau média (12). En outre, la relation entre la marque et le consommateur semble grandement affectée par l'utilisation d'Internet qui permet une communication plus rapide, continue, interactive et personnalisée.

Deux principales stratégies de marque s'offrent à toute entreprise désirant être présente sur Internet. La première correspond à l'utilisation d'une marque existant déjà dans le monde réel et employant alors Internet comme un nouveau moyen de communication et/ou comme un nouveau canal de distribution. On parle alors de «click-and-mortar » : ces entreprises sont présentes à la fois dans l'économie traditionnelle et sur Internet via un site portant leur nom. La seconde stratégie consiste à créer une nouvelle marque qui n’existe que dans le monde virtuel : la e-marque. L'objectif de cet article est précisément de s'intéresser au seul cas des emarques créées sur Internet et à leurs spécificités. De nombreuses questions se posent alors. Quel est le type de relation qui se développe entre les internautes et les e-marques ? Ces marques apportent-elles de la valeur ajoutée au service proposé ? Enfin, ces marques peuventelles construire un fort capital-marque?

Pour répondre à ces interrogations, la présente recherche étudie dans un premier temps les différents types de e-marques sur Internet. Les résultats d'une étude exploratoire, portant sur la perception des e-marques par les consommateurs, sont ensuite exposés. Enfin, cette 
recherche suggère aux entreprises des pistes de réflexion pour la construction du capitalmarque de leurs e-marques.

\section{LES DIFFERENTS TYPES DE E-MARQUES}

D'un point de vue historique, les premières marques présentes sur le réseau mondial au début des années 1990 se rapportent "génétiquement » aux activités liées à Internet : il s'agit de moteurs de recherche, de fournisseurs d'accès, etc. Ces marques sont nées sur Internet par la création d'un site spécifique (Club Internet, World On Line, Yahoo, Altavista, etc.). Aujourd'hui, les créations de nouvelles marques sur le Net concernent la catégorie des sites de vente en ligne comme Cdiscount, Alapage, Lastminute, Easycar, etc. L’intérêt de développer une e-marque réside dans la construction d'une identité propre, sans image de marque initiale à protéger. Ceci permet de définir une marque adaptée à l'univers d’Internet et aux nouveaux services proposés.

\section{Encadré 1 : Définition d'une e-marque}

Les e-marques sont des marques créées et présentes exclusivement sur Internet : l'accès à leurs produits ou à leurs services se fait uniquement via le média Internet, par l'intermédiaire d'un site portant le nom de la marque.

Selon cette définition, les e-marques constituent un ensemble très hétérogène et complexe qu'il convient de structurer afin de préciser les spécificités de la gestion des e-marques sur Internet. Dans ce but, deux critères semblent particulièrement pertinents ici : les e-marques peuvent être distinguées selon la présence ou non de la caution d'une marque «brick-andmortar » (e-marque cautionnée ou autonome) et selon le type de site qu'elles développent (emarque associée à un site transactionnel ou à un site informationnel). 
Les e-marques cautionnées ou autonomes

Les e-marques cautionnées correspondent à la création de nouvelles marques sur Internet cautionnées par une entreprise de l'économie traditionnelle. Par exemple, Ooshop est une emarque cautionnée par le distributeur Carrefour, dont le nom apparaît clairement sur le site web, ainsi que dans toutes les communications commerciales. L'objectif premier est de développer une marque possédant une identité propre et un nom adapté à l’univers d’Internet, tout en bénéficiant du capital-marque de la marque-mère. Celle-ci sert de caution et de garantie pour instaurer un rapport de confiance avec le consommateur, qui sait précisément quelle entreprise est à l'origine de la e-marque.

Les e-marques autonomes correspondent à la création de nouvelles marques sur Internet, sans aucune caution ou sans aucune référence visible à l'économie traditionnelle. Ces marques sont généralement créées par les acteurs émergents de la nouvelle économie (Ebay, Easycar, etc.). Toutefois, les e-marques autonomes peuvent également être développées par des entreprises de l'ancienne économie, ceci pour deux raisons principales. Premièrement, le nom de la société existante peut n’apporter aucune plus-value par rapport à l'offre concurrente. Deuxièmement, l'entreprise existante ne veut pas prendre le risque d'afficher son nom avec celui de la e-marque par peur de retombées négatives, notamment dans le cas d'un échec. Par exemple, Reflect est une nouvelle marque de cosmétique vendue exclusivement en ligne (sur le site « Reflect.com ») par le groupe Procter \& Gamble qui ne mentionne pas son nom dans la commercialisation de cette nouvelle marque.

\section{INSEREZ TABLEAU 1}

Les e-marques associées à un site transactionnel ou à un site informationnel

Les e-marques associées à des sites informationnels offrent gratuitement un service. C'est notamment le cas des moteurs de recherche (Yahoo, Altavista, Google, etc.), des portails 
(Tiscali, Free, etc.) ou encore des sites thématiques (Aufeminin, Bebe, etc.). Les e-marques rattachées à des sites transactionnels vendent en ligne des produits ou des services (Telemarket, Lastminute, Alapage, etc.).

Quel que soit le type de e-marque, le développement d'une marque sur Internet implique des risques importants. La construction d'une e-marque s'opérant ex nihilo sur un nouveau média, la principale difficulté est de ne pas réussir à construire un véritable capital-marque, avec une image de marque précise, des associations fortes, une forte notoriété et des clients fidèles. La gestion des e-marques sur Internet représente donc un véritable défi managérial et mérite une réflexion spécifique sur les outils de gestion qui leur sont les plus adaptés. En effet, mis à part le développement de leur offre produit/service et l'utilisation de quelques supports de communication, ces marques naissent et vivent uniquement au travers d'Internet. Comment ces marques peuvent-elles alors créer un capital-marque ? Comment les consommateurs perçoivent-ils ces marques ? La réponse à ces questions nécessite un travail exploratoire qui prend la forme d’une étude qualitative portant sur l'évaluation des e-marques par les internautes.

\section{LA PERCEPTION DES E-MARQUES : ETUDE QUALITATIVE AUPRES DES CONSOMMATEURS}

La marque n’a de raison d'exister que si elle apporte de la valeur aux consommateurs. Le concept de capital-marque correspond précisément à la valeur ajoutée d’une marque associée à un produit ou à un service (6). 


\section{Cadre théorique : le capital-marque d'une e-marque}

Le concept de capital-marque du point de vue du consommateur constitue le cadre d'analyse de l'étude qualitative. Selon cette approche, une marque à fort capital correspond à une marque qui apporte de la performance supplémentaire au produit ou au service marqué aux yeux des consommateurs (5). Cette valeur ajoutée est le résultat de tous les investissements marketing engagés sur la marque (10). Le capital-marque se construit donc sur le long terme. Dans le cadre de cette recherche, nous avons retenu l'approche consommateur du capitalmarque, qui est alors étudié selon les perceptions et les comportements des individus (15). Pour évaluer le capital-marque, nous nous appuyons plus particulièrement sur les dimensions suivantes : la notoriété, la fidélité des consommateurs, la force de l’image de marque et la force des signes de reconnaissance (1).

\section{Méthodologie de l'étude qualitative exploratoire}

Pour cerner la perception des e-marques, une étude qualitative a été réalisée, sur la base d'entretiens individuels en utilisant un guide d'entretien. Ce mode de collecte de l'information se justifie par le caractère exploratoire de cette recherche, et surtout par le type de données à collecter : les comportements de navigation sur Internet, la perception des sites et des marques par les consommateurs, etc. Seize entretiens semi-directifs ont ainsi été menés auprès d'internautes âgés de 18 à 50 ans. Cet échantillon, sans pour autant être représentatif de la population des internautes français, recouvre à la fois des personnes encore peu familiarisées avec Internet et des internautes confirmés n'hésitant pas à faire leurs achats via ce moyen de distribution. Les personnes interrogées ont été amenées à s'exprimer sur plusieurs e-marques et en particulier sur celles qu'elles connaissaient le mieux. Les seize entretiens ont été intégralement retranscrits et une analyse de contenu thématique a été réalisée à partir de ce matériau. 


\section{Résultats de l'étude qualitative}

L’étude qualitative met en évidence trois principaux résultats quant à la perception des emarques par les internautes : une image peu précise, une forte notoriété et fidélité, une image exclusivement utilitaire pour les e-marques transactionnelles et une image plus hédonique pour les e-marques informationnelles.

\section{L’image peu précise des e-marques}

La première idée qui émerge très nettement de l'étude exploratoire est la grande difficulté qu'éprouvent les consommateurs interrogés à parler des e-marques. D'une manière générale, ils ont du mal à s'exprimer sur les e-marques, y compris sur celles qu'ils connaissent pourtant bien, et dont ils fréquentent régulièrement le site Internet.

«Hourra.fr, je n'en ai pas d'idées précises ; c'est encore trop flou, trop neuf, même si je m’en sers régulièrement. ».

«Amazon ou Ooshop, je connais bien les sites, mais il m'est difficile de vous en parler, d'avoir une image précise ; c'est abstrait. »

Ainsi, même si certaines marques ont acquis une très forte notoriété sur Internet, leurs valeurs ne sont pas clairement perçues par le consommateur. Les associations qui leur sont attribuées sont assez rares ; elles sont de surcroît très générales. Le plus souvent, les images énoncées par les individus interrogés sont associées au média Internet en général, et non pas à la marque en particulier. Des associations comme "moderne », «technologique », « dynamique », « nouveau », « adaptation », « innovation » sont les plus fréquemment citées et elles sont liées à de nombreuses e-marques, qu’il s’agisse de moteurs de recherche (Yahoo ou Google) ou bien de sites de vente en ligne (Amazon ou Alapage). Le caractère général des associations ne permet pas aux e-marques d'affirmer clairement leur positionnement. Ceci constitue alors un obstacle fondamental à la construction de leur capital-marque, dans la 
mesure où le capital d'une marque repose entre autres sur des images fortes et spécifiques, c’est-à-dire indépendantes du secteur d'activité de la marque.

L'étude qualitative a permis d'expliquer en partie la difficulté des consommateurs à associer des images fortes aux e-marques. La raison principale est le manque de supports matériels de ces marques créées uniquement dans le monde virtuel. Les signes de reconnaissance des emarques sont peu visibles ; elles disposent de moyens d'expression limités pour construire leur capital-marque. Les seuls points d'ancrage et de contact avec le consommateur sont le site Internet, la communication et le service offert (l'offre produit et la logistique, dans le cas d'un site marchand).

«Je n’ai qu’une relation lointaine avec Ooshop, une relation épisodique, uniquement quand je me connecte et quand je réceptionne mes commandes. C'est très limité, et ça n’a rien à voir avec ma relation avec le Monoprix au coin de ma rue. »

«Un site Internet, c'est très abstrait. J'ai acheté un voyage sur Travelprice, très rapidement, sans parler à personne, sans aucun conseil. Du coup, on ne peut même pas dire que je connais bien Travelprice. »

Une autre raison pouvant expliquer les difficultés du consommateur à s'exprimer sur les emarques est la présence de nombreuses marques de type «me-too », l’innovation d'un site pouvant être très rapidement copiée. Les «me too » sont alors des déclinaisons d'une idée existante ; elles ne présentent pas de réelle innovation et ne permettent pas une différenciation très nette par rapport à la concurrence. De ce fait, les «me-too » souffrent de problèmes de mémorisation de la part du consommateur, et ont rarement un positionnement clair par rapport aux sites concurrents. Par exemple, un consommateur utilisant plusieurs sites immobiliers (Immostreet, Avendre, Entreparticuliers) estime que ces sites se ressemblent tous. 
«Même si l'ergonomie du site est différente, ils apportent tous le même service de base ; on peut faire les mêmes critiques. Il n'y a pas assez de différences pour qu'un site sorte du lot».

«Pour acheter un CD sur Internet, il n'y a pas de solution miracle, c'est-à-dire pas de site miracle. Tous les sites comme Alapage vont me proposer le même disque, au même prix et dans les mêmes conditions de livraison. »

Lors des entretiens individuels, les consommateurs comparent souvent, de façon très spontanée, l’image des e-marques à celle des «click-and-mortar». Il apparaît ainsi que le contact entre la e-marque et le consommateur est bien différent de celui existant avec les marques traditionnelles qui peuvent être portées par de plus nombreux éléments tangibles : le packaging des produits, les catalogues, les points de vente, les vendeurs, etc. L'analyse qualitative montre alors que les internautes interrogés ont nettement moins de difficultés pour s’exprimer sur les marques de type «click-and-mortar» comme Darty ou la Fnac. Dans ce cas, les associations à la marque sont plus nombreuses et surtout plus précises. Le consommateur se réfère avant tout au positionnement traditionnel et aux signes de reconnaissance développés dans le monde réel.

«Au moins pour évaluer le site de la Fnac, on a des points de repère précis, c'est-àdire les magasins que je connais bien ; ce n'est pas le cas pour Amazon.fr ; Fnac.com sur Internet, pour moi, c'est un large choix, plus large qu'en magasin, avec toujours les réductions sur les nouveautés et des avantages pour les adhérents. »

Pour évaluer une «click-and-mortar», l’individu procède par analogie avec la marque bien connue dans le monde réel. Toutefois, le développement d'une «click-and-mortar» sur 
Internet fait également apparaître de nouvelles associations qui la font évoluer et qui justifient par conséquent son arrivée sur le Net.

« Le site de la SNCF est maintenant un outil indispensable pour moi ; s'ils ne l'avaient pas fait ça aurait été la preuve de leur incapacité à s'adapter aux nouvelles technologies. »

«Avec leur site Darty.fr, on sait maintenant qu'on est face à une entreprise moderne, à la pointe, qui nous apporte du confort, une facilité d'achat, à domicile. »

\section{Une forte notoriété et fidélité aux e-marques}

Sans que cela ait pu être précisément mesuré, l'étude qualitative montre que de nombreuses emarques ont su profiter de la « vague Internet » et développer rapidement leur notoriété. Mais, l'étude met surtout en valeur une très forte fidélité des consommateurs aux e-marques. En ce qui concerne les sites transactionnels, le consommateur semble avoir des habitudes très rigides. En dépit de la facilité d'accès aux autres sites concurrents sur Internet, il ne change pas facilement de distributeurs. Ceci est particulièrement vérifié pour les sites de vente en ligne comme Hourra ou Ooshop.

«Maintenant que j’ai choisi Hourra, mes courses sont mémorisées, et je continue avec Hourra, c'est plus simple. [..] Je pourrais aller voir ailleurs, aller voir les concurrents. En quelques clicks, ce n'est pas difficile. »

«J'achète tous mes disques et tous mes livres sur Fnac.com. Je suis très fidèle. Je ne vais pas voir ailleurs maintenant que je connais très bien leur site et son fonctionnement. »

Dans le cas des e-marques associées à un site informationnel, on retrouve également une forte fidélité de l’internaute. 
«J'ai l'habitude de me connecter très régulièrement sur le portail Voila.fr, en fait à chaque fois que je suis sur Internet ou presque. J'y retrouve toutes les informations, l'actualité, les informations pour mes sorties au ciné, les résultats sportifs aussi quand ça m'intéresse. C'est comme consulter son journal, son quotidien ou son magazine préféré. »

«Mes sites préférés, ce sont ceux qui concernent le cinéma et le rugby, mes deux passions. Je les ai mis en favoris. Et je les consulte tout le temps, presque autant que ma messagerie. »

\section{La dominante utilitaire des e-marques transactionnelles et la dominante hédonique des e-marques informationnelles}

Une autre idée majeure mise en évidence par les entretiens individuels est l'opposition très nette entre la dimension utilitaire attachée aux e-marques transactionnelles et la dimension hédonique attachée aux e-marques informationnelles. En effet, on observe très clairement des attitudes, des motivations et des comportements différents selon ces deux principaux types de sites web.

Les e-marques rattachées aux sites marchands sont associées à une idée de praticité, à une motivation utilitaire. Pour le consommateur, ces marques correspondent à des outils fonctionnels et utilitaires : l'internaute recherche des informations sur une offre produit, ou bien il cherche à réaliser la meilleure transaction possible. Par conséquent, les rares associations données par les consommateurs interrogés au sujet des e-marques transactionnelles reposent avant tout sur la notion de fonctionnalité du site: «confort », «efficace ", "facilite la vie pour acheter à distance", "pour faire le meilleur achat possible », " pratique à utiliser », etc. 
Dans ce cas, le consommateur a généralement une idée bien précise, et par conséquent un comportement de navigation limité. Il ne cherche pas à découvrir de nouveaux produits ; il ne fait pas véritablement de shopping sur Internet.

«Quand j'achète sur Internet, je sais ce que je veux ; je sais que je peux l'acheter sur tel site, et alors, je me connecte juste pour faire cet achat précis. »

«Internet, ce n'est pas vraiment le bon endroit pour faire du shopping, pour flâner et découvrir ; et pour cela, je préfère faire les boutiques et avoir les produits en face de moi. »

«Pour acheter sur Internet, c'est simple, je vais droit au but. Je passe ma commande le plus vite possible, et je me déconnecte. »

Dans ces conditions, lors de visites de sites transactionnels, le consommateur semble rarement cliquer sur les rubriques annexes, ou sur les bandeaux publicitaires. Ainsi, la dimension plaisir ou hédonique semble quasiment absente de l'achat sur Internet, ce qui représente un obstacle pour les e-marques transactionnelles dans la construction de leur capital-marque.

« Je ne prends aucun plaisir à surfer sur Internet quand je fais mes achats, je l'utilise pour me ravitailler ; plus c'est pratique, mieux c'est. »

« Je n'ai pas le temps de m'attarder sur le site, de voir ce qu'il propose vraiment, de voir ce que fait l'entreprise et qui elle est vraiment. »

En revanche, les e-marques informationnelles semblent avoir une forte dimension hédonique, en plus de la dimension utilitaire non négligeable dans ce cas. Généralement, le consommateur va consulter un site informationnel en relation avec ses centres d'intérêt ; il est donc plus enclin à avoir un comportement de navigation exploratoire et à découvrir de nouvelles informations ou de nouveaux produits. 
«J'ai tendance à ne pas cliquer sur les autres rubriques sauf pour les sites qui correspondent vraiment à mes centres d'intérêt, à mes loisirs. »

L'étude qualitative montre que c'est lors de la visite de ces sites informationnels, à la recherche d'informations relatives à ses passions, que le consommateur semble prendre le plus de plaisir, en profitant de conseils ou en échangeant avec d'autres internautes. Le consommateur est le plus enclin à surfer, c'est-à-dire à cliquer, par simple curiosité, sur toutes les rubriques d'un site, et à passer, au gré de ses humeurs, d'un site à un autre via les liens hypertexte.

«Quand je recherche des informations sur un thème qui me passionne, ça me prend du temps ; je peux y passer des heures car c'est un véritable plaisir. » «Si je surfe autour d'un thème qui me plait, c'est pour apprendre, pour être surprise, pour découvrir, pour partager aussi avec d'autres personnes qui ont les mêmes centres d'intérêt. »

Ainsi, l’étude exploratoire montre que, malgré une notoriété et une fidélité qui peuvent être très fortes, les difficultés des e-marques sont particulièrement saillantes en ce qui concerne la force de l’image de marque et la puissance des signes de reconnaissance. Ces résultats nous amènent à définir certaines implications managériales pour la construction du capital-marque des e-marques. 


\section{IMPLICATIONS MANAGERIALES}

Pour construire des e-marques fortes et durables, il est important de définir leur identité, leur mission et de les communiquer. Par ailleurs, le succès des e-marques repose sur leur capacité à acquérir la confiance des internautes et à fidéliser les e-consommateurs.

\section{Définir l'identité de la e-marque}

Afin de construire une image forte, les e-marques sont amenées inévitablement à définir et à communiquer des valeurs fortes. Ces valeurs doivent être spécifiques à la marque et se détacher du support d’Internet pour devenir des éléments de différenciation. Des marques comme Alapage ou Ooshop rencontrent, par exemple, des difficultés à développer des images fortes et sont peu rattachées à des traits de personnalité. En effet, la e-marque Ooshop, associée à une image "moderne », "pratique » et «utile», se différencie peu de ses concurrents. De même, la marque Alapage, rattachée aux associations « diversité de l’offre », «fonctionnel» et «nouvelle technologie », se distingue peu de l'image d'Internet. A l'inverse, une e-marque comme Reflect, proposant des produits cosmétiques sur mesure, se différencie de ses concurrents et s’efforce de communiquer sur cette valeur distinctive. Pour jouer leur rôle de différenciation, les e-marques peuvent s'appuyer sur des éléments fonctionnels, symboliques et expérientiels.

\section{- Bénéfice fonctionnel}

Sur Internet, la relation entre l'internaute et la marque repose principalement sur les caractéristiques fonctionnelles, c'est-à-dire sur la qualité de l'offre produit ou service. Les marques qui s’appuient sur un bénéfice fonctionnel, par exemple «Lastminute.com, les voyages et les loisirs de dernière minute », « Allocine.fr, l’information cinématographique », « Prenoms.com, le guide des prénoms » se différencient indéniablement de la concurrence en affichant un positionnement clair et fort. Ces éléments de différenciation sont particulièrement 
efficaces dans l'univers d'Internet où l'internaute est en général à la recherche d'informations, de produits ou de services précis.

- Bénéfice symbolique

Il est encore difficile aujourd'hui pour les e-marques de se différencier sur des éléments symboliques. Certaines e-marques cherchent à se démarquer des concurrents par la création de noms qui n'expriment pas leur fonction, mais visent à revendiquer un certain symbolisme comme Amazon (librairie), Houra (supermarché), Yahoo (portail) ou encore Opodo (agence de voyages). Toutefois, ces e-marques sont davantage associées à une mission précise et sont donc surtout rattachées à des caractéristiques fonctionnelles. L’étude exploratoire révèle, en effet, que les e-marques connaissent un déficit au niveau des bénéfices symboliques. Il est pourtant important pour les e-marques de développer ces éléments de différenciation symboliques qui ont une répercussion sur leur évaluation (2).

- Bénéfice expérientiel

La relation entre l'internaute et la e-marque repose également sur l'expérience ressentie par l'internaute lors de sa visite sur le site (8). Il est donc essentiel pour les e-marques de définir avec soin l'aspect général et l'ergonomie du site, la qualité de navigation, le processus de traitement des commandes et l'utilisation d'une technologie de pointe. Les différents points de contact avec l'internaute sont également des éléments essentiels à l'évaluation du bénéfice expérientiel. En offrant un contact personnalisé et humain aux internautes, par le biais d’un centre d'appels utilisant téléphone fixe, mobile, mail, Web, SMS, les e-marques des sites transactionnels peuvent améliorer le bénéfice expérientiel de leur clientèle et accroître le nombre d'acheteurs parmi les visiteurs.

\section{Exprimer l’identité de la marque}

Pour traduire son identité de façon concrète, une e-marque peut mener une communication 
on-line et off-line et mettre en place des alliances de marques.

- Mener une communication on-line et off-line

Les signes de reconnaissance jouent un rôle primordial dans la compréhension de la marque. Ainsi, les e-marques, affichant un logo, des couleurs, une signature revendiquant leur mission, ont plus de chance d'avoir une image claire que celles qui se limitent dans l'expression de leurs valeurs. L’introduction d'une e-marque dans le monde réel, en proposant un nouveau contact au consommateur, peut renforcer sa relation avec le client et ses signes de reconnaissance. C’est ainsi que la plupart des vépécistes ouvrent des points de vente ou des relais services afin d’assurer une relation privilégiée avec leur clientèle, mais également afin d'attirer de nouveaux consommateurs. Par ailleurs, pour exprimer son identité, la communication des e-marques doit certes s’appuyer sur le média on line, mais elle ne doit pas négliger les média classiques (affichage, presse, etc.). « Netclub.fr » vente ainsi son club de rencontre au travers de messages publicitaires télévisés. La e-marque doit alors veiller à tenir le même discours on line et off line.

- Développer les partenariats entre e-marques

Les alliances entre e-marques représentent un autre moyen pour revendiquer les valeurs de la e-marque. La complémentarité des marques ou l'association des marques autour d’un univers commun permet de créer une image claire et forte dans l'esprit des consommateurs (3). Les alliances entre e-marques peuvent s’illustrer par la création d'un site commun, et par la vente ou la communication inter-sites (Tableau 2).

\section{INSEREZ TABLEAU 2}

Afin d'apporter une valeur ajoutée aux consommateurs, il est important de créer une logique d’affinité et de fédérer les marques autour d'un univers qui reflète leur offre. Un site commun tel que « Mygarden.com » est un véritable succès. Il s’adresse à des passionnés de jardinage. 
Il est alors tout aussi important pour les marques de ce secteur d’être présent sur ce site, qui fédère tous les clients potentiels, que de posséder leur propre site. L’internaute bénéficie ainsi d’une information plus complète sur un centre d’intérêt spécifique et profite des compétences et du service des marques regroupées autour d’un univers particulier.

Afin de traduire l’identité d’une e-marque, il est également essentiel de l’insérer sur des sites qui partagent des valeurs communes. Ainsi, «Amazon.fr », s’associant avec des sites qui présentent et commentent des ouvrages liés à leur thématique (bandes dessinées, base-ball, peinture...), cherche à communiquer la diversité de ses produits. «Eveiletjeux.com », s’alliant avec des sites concernant l'univers des enfants et des mamans («Bebe.fr», « Maman.fr », « Aufeminin.com »), vise à s’intégrer dans l’univers de ses consommateurs potentiels. Des liens hypertextes de Microsoft, Adobe, Canon, Apple, Compaq sont présents sur le site «Jungle.com» qui commercialise du matériel informatique. Ces opérations engendrent une meilleure visibilité, ainsi qu'un contact direct avec la marque « invitée ».

Par ailleurs, les alliances de marques permettent d'enrichir l'image des marques alliées (4). La présence d'une marque de luxe sur un site de golf permet, par exemple, de fortifier et de nourrir les deux marques. Le choix des marques associées doit s’inscrire dans la définition d'un positionnement clair et dans une cohérence d'image afin de ne pas diluer l'image des marques.

\section{Acquérir la confiance des internautes}

La confiance dans la marque est un élément sous-jacent à l'établissement d’une marque forte. La confiance dans la marque peut se traduire par la crédibilité de la marque, son intégrité et sa bienveillance envers les consommateurs (9). 
- Sécuriser le consommateur

Dans le domaine du commerce électronique, la confiance envers la e-marque repose, entre autre, sur le paiement sécurisé et le respect de la vie privée (7). Il est donc très important pour les e-marques de mettre en avant leur démarche déontologique, en exposant leur politique de respect de la vie privée. Les marques nées sur Internet commencent souvent par communiquer à l'internaute, leurs coordonnées postales comme pour «matérialiser » leur existence. L'utilisation de logos d'organismes certificateurs représente également une source de confiance (11). La plupart des sites des e-marques transactionnelles intègrent, d'ailleurs, aujourd’hui un ou plusieurs logos destinés à générer la confiance de l’internaute. Ainsi les logos des grands organismes bancaires, tels que Visa ou American Express, visent à rassurer l’acheteur sur la sécurité de paiement.

- Introduire un marketing relationnel

Il est plus difficile pour une e-marque d'acquérir la confiance des consommateurs que pour une marque «brick-and-mortar » qui détient déjà un capital confiance important dans le monde off-line. La confiance d'une e-marque repose donc essentiellement sur une démarche relationnelle. Lorsqu'une e-marque cherche à acquérir la confiance de ses internautes, il peut être judicieux de faire appel à des témoignages de garantie (11). Ainsi, certains sites, comme «Ebay.com » (encadré 2) ont attiré de nombreux acheteurs du monde entier et peuvent aujourd'hui se servir de ce portefeuille clients comme une source de confiance. Les sites communiquent d'ailleurs ouvertement sur le nombre et les réactions des acheteurs déjà conquis. Il peut être également judicieux pour une e-marque d’être référencée sur un portail reconnu qui pourrait lui apporter une certaine crédibilité et être générateur de trafic. La notoriété et l’image du portail comme Yahoo ou Aol, bénéficient implicitement aux sites qu’il présente, ce qui constitue un facteur de confiance pour le visiteur internaute. La e-marque 
peut ainsi acquérir la confiance des internautes à travers le développement d’un marketing relationnel.

\section{Fidéliser les e-consommateurs}

Pour fidéliser leur clientèle, les e-marques peuvent s’appuyer sur les échanges interpersonnels et créer des marques communautaires mais elles doivent surtout offrir un produit ou un service de qualité sans cesse renouvelé.

- Créer des e-marques communautaires

Pour fidéliser les individus les e-marques doivent favoriser les échanges entre les internautes et la marque, en introduisant, par exemple, des conseils de consommation relatifs à leurs produits ou leurs services. Il est également important de soigner les échanges interpersonnels entre internautes qui permettent de fédérer les individus autour de valeurs communes rattachées à la e-marque. C’est ainsi que se construisent des marques fortes autour de communautés sur Internet. Ces marques communautaires sont créées selon une logique d'affinités autour d'une marque ou plus largement autour de l'univers d'une marque (14). Par le biais notamment des forums de discussion, les e-marques s'efforcent ainsi de favoriser les échanges interpersonnels qui induisent un certain attachement et par conséquent une certaine fidélité. Cette expérience correspond à une stimulation sensorielle ou environnementale qui émane de la qualité des échanges interpersonnels entre les internautes. La relation à la marque peut alors être symbolisée par une triade entre les consommateurs et la marque (13).

INSEREZ FIGURE 1 
- Renouveler l’offre

La e-marque doit développer et apporter continuellement un service enrichi à ses consommateurs, c’est-à-dire des services à réelle valeur ajoutée, renouvelés et améliorés. Sinon, le site risque de voir ses internautes se tourner vers des concurrents dont l'innovation serait plus attrayante. Ainsi «Parisétudiant.com » cherche à rendre son site évolutif par la participation des internautes sur chacun des sujets traités. Mais la fidélisation aux e-marques dépend également de la transparence, de l'ergonomie du site et de sa réactivité, et par conséquent de la qualité du service et des produits.

\begin{tabular}{l}
\hline Encadré 2 : Le développement d'une e-marque, l'exemple Ebay \\
\hline 1- Définition de l'identité \\
La mission d'ebay est de relier en temps réel les acheteurs et vendeurs du monde entier et de \\
leur permettre d'échanger en direct les objets venus des quatre coins du monde. Le \\
mécanisme des enchères, permettant à l'objet vendu d'atteindre son meilleur prix, représente \\
un élément de différenciation par rapport aux concurrents qui pratiquent pour la plupart des \\
prix fixes. \\
2- Expression de l'identité \\
Si ebay est une mine de bonnes affaires, le site cherche aussi à être un espace de \\
communication et de rencontre où chacun doit être traité avec le plus grand respect. Ebay \\
s'efforce donc de développer un éventail de rubriques et de services pour que les utilisateurs \\
puissent réaliser efficacement des affaires en toute sécurité. La e-marque est devenue une \\
partie intégrante de la culture américaine. On en parle dans les films, elle fait l'objet de livres, \\
d'articles, etc. \\
3- Acquisition de la confiance des internautes \\
La qualité de l'offre est assurée par la qualité des membres les plus actifs sur le site. Le \\
fonctionnement du site se fonde sur la confiance que les internautes s'accordent entre eux. Ce \\
qui est important c'est que les vendeurs et les acheteurs soient assurés d'une transaction sûre \\
et honnête. \\
4- Fidélisation des internautes \\
La fidélisation d'ebay réside dans la création d'une marque communautaire. Ebay s'efforce de \\
laisser ses membres faire vivre le site, par le biais du forum de discussion, de la \\
personnalisation des annonces, etc. Ebay veut que ses membres éprouvent un sentiment \\
d'appartenance à une communauté. Les collectionneurs, passionnés des enchères, restent ainsi \\
la cible privilégiée du site. Les collectionneurs assurent, en effet, le jeu de l'offre et de la \\
demande et accentuent le phénomène du "bouche à oreille ». En outre, la force de la \\
communauté d'ebay se trouve dans son étendue mondiale qui offre un élément distinctif \\
indéniable par rapport à la concurrence.
\end{tabular}




\section{CONCLUSION}

La présentation des différentes stratégies de marque sur Internet laisse apparaître que la gestion des e-marques est spécifique et qu’une problématique majeure de ces marques concerne la construction de leur capital-marque. Les e-marques souffrent d'une image peu précise et de signes de reconnaissance peu développés. Il apparaît également une nette opposition entre les e-marques informationnelles qui possèdent une dominante hédonique (même si elles rendent un service au consommateur et ont une forte valeur utilitaire) et les emarques transactionnelles à dominante utilitaire. En revanche, les e-marques bénéficient de consommateurs fidèles, malgré la facilité qu’offre le média Internet pour aller tester la concurrence.

Pour remédier aux limites des e-marques, il est essentiel de définir précisément leur identité, leur mission. La définition des valeurs des e-marques, en amont, permettra ainsi de communiquer de façon cohérente et forte auprès des différentes cibles. Par ailleurs, les emarques doivent s’efforcer d'acquérir la confiance des internautes et de ne pas délaisser les actions de fidélisation auprès de leurs clients. L’article apporte, à cet égard, des recommandations afin que les entreprises manipulent au mieux ces différents facteurs clés de succès. Toutefois, la gestion des e-marques, par la spécificité du média sur lequel elles évoluent, mériterait de se fonder sur des outils adaptés à ce type de marque. Il serait, par exemple, opportun de développer des outils de mesure spécifiques à l'évaluation de la relation entre l’internaute et la e-marque, ou encore spécifiques à la mesure de l'image de marque, en évaluant l’importance des bénéfices fonctionnels, symboliques et expérientiels. 


\section{BIBLIOGRAPHIE}

(1) Aaker D.A. (1991), Managing brand equity, New York, The Free Press.

(2) Bhat S. et Reddy K. (1998), Symbolic and functional positioning of brands, Journal of Consumer Marketing, 15,1, 32-41.

(3) Boad B. (1999), Co-branding opportunities and benefits, in Co-branding, the Science of Alliance, Blackett T. et Boad B, New York, St. Martin’s Press, 22-37.

(4) Cegarra J.J et Michel G. (2001), Co-branding : clarification du concept, Recherche et Applications en Marketing, 16, 4, 57-69.

(5) Changeur S. (1999), Le territoire de marque : proposition et test d'un modèle basé sur la mesure des associations des marques, Thèse de Sciences de Gestion, IAE d'Aix-Marseille.

(6) Farquhar P.H. (1990), Managing brand equity, Journal of Advertising Research, 30, 4, RC7-RC12.

(7) Gauzente C. (2003), Vie privée et Internet: la pratique des sites français, Décisions Marketing, 30, 7-17.

(8) Gonzalez C. (1999), Impact of perceived legibility and perceived stimulation of electronic catalogue on consumer satisfaction, $12^{\text {th }}$ EMAC Doctoral Colloquium, Humbolut University, Berlin, 9-11 mai 1999.

(9) Gurviez P. et Korchia M. (2002), Proposition d'une échelle de mesure multidimensionnelle de la confiance dans la marque, Recherche et Applications en Marketing, 17, 3, 41-59.

(10) Keller K.L. (1998), Strategic brand management, Upper Saddle River NJ, Prentice Hall.

(11) Lehu J.M. (2001), Stratégie de marque.com, Paris, Editions d’Organisation.

(12) Lévy J. (2000), Impact et enjeux de la révolution numérique sur la politique d'offre des entreprises, Revue Française du Marketing, 177/178, 13-28. 
(13) McAlexander J.H., Schouten J.W. et Koenig H.F. (2002), Building brand community, Journal of Marketing, 66, 1, 38-54.

(14) McWilliam G. (2000), Building stronger brand through online communities, Sloan Management Review, 41, 3, 43-54.

(15) Park C.S et Srinivasan V. (1994), A survey-based method for measuring and understanding brand equity and its extentibility, Journal of Marketing Research, 31, 2, 271-288. 


\begin{tabular}{|c|l|l|l|}
\hline $\begin{array}{c}\text { Stratégie de } \\
\text { marque }\end{array}$ & \multicolumn{1}{|c|}{ Objectifs } & \multicolumn{1}{c|}{ Risques } & \multicolumn{1}{c|}{ Exemples } \\
\hline $\begin{array}{c}\text { E-marque } \\
\text { cautionnée }\end{array}$ & $\begin{array}{l}\text { - Développer une marque } \\
\text { avec une identité propre et } \\
\text { bénéficier du capital de la } \\
\text { marque caution } \\
\text { - Attirer une nouvelle } \\
\text { clientèle vers la marque } \\
\text { caution }\end{array}$ & $\begin{array}{l}\text { - Dilution de la } \\
\text { marque caution qui } \\
\text { s'étend vers de } \\
\text { nouveaux territoires }\end{array}$ & $\begin{array}{l}\text { ooshop.com (Carrefour) } \\
\text { autovalley.fr (BNP/Paribas) } \\
\text { hotmail.com (Microsoft) }\end{array}$ \\
\hline $\begin{array}{l}\text { E-marque } \\
\text { autonome }\end{array}$ & $\begin{array}{l}\text { - Créer une marque avec } \\
\text { une identité propre, } \\
\text { adaptée à Internet }\end{array}$ & $\begin{array}{l}\text { - Difficulté à } \\
\text { émerger, à se } \\
\text { démarquer sur le } \\
\text { média Internet }\end{array}$ & $\begin{array}{l}\text { reflect.com } \\
\text { amazon.com } \\
\text { cdnow.com } \\
\text { lastminute.com } \\
\text { ebookers.com } \\
\text { easycar.com }\end{array}$ \\
\hline
\end{tabular}

Tableau 1 : Création de nouvelles marques sur Internet 


\begin{tabular}{|c|l|l|}
\hline & \multicolumn{1}{|c|}{ Caractéristiques } & \multicolumn{1}{c|}{ Objectifs } \\
\hline $\begin{array}{c}\text { Création d'un } \\
\text { site commun }\end{array}$ & $\begin{array}{l}\text { Collaboration entre différentes } \\
\text { marques d'un même secteur } \\
\text { pour créer un site sur l'univers } \\
\text { des marques associées }\end{array}$ & $\begin{array}{l}\text { - Fédérer les marques autour d'un } \\
\text { univers qui renforce leur image }\end{array}$ \\
\hline $\begin{array}{c}\text { Vente et } \\
\text { communication } \\
\text { inter-sites }\end{array}$ & $\begin{array}{l}\text { Coopération entre marque } \\
\text { invitée et marque d'accueil, à } \\
\text { travers des liens inter-sites. }\end{array}$ & $\begin{array}{l}\text { - Augmenter la visibilité des } \\
\text { marques } \\
\text { - Enrichir les marques en les } \\
\text { associant à des thèmes en } \\
\text { cohérence avec leur mission, leur } \\
\text { image. }\end{array}$ \\
\hline
\end{tabular}

Tableau 2 : Stratégie d'alliances de marques sur Internet 


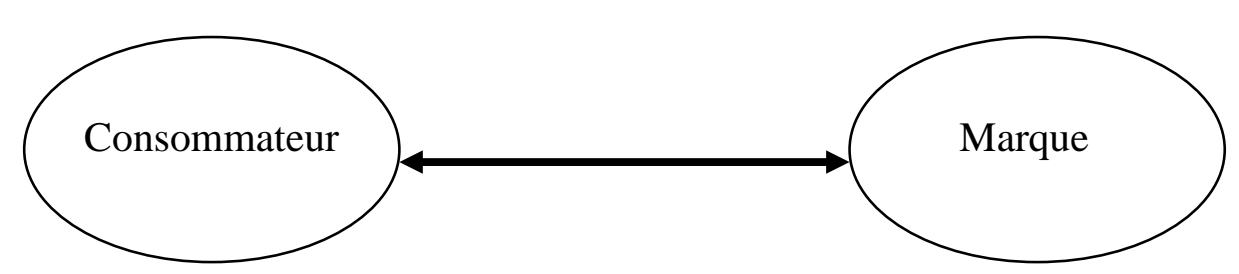

Modèle traditionnel de la relation entre marque et consommateur

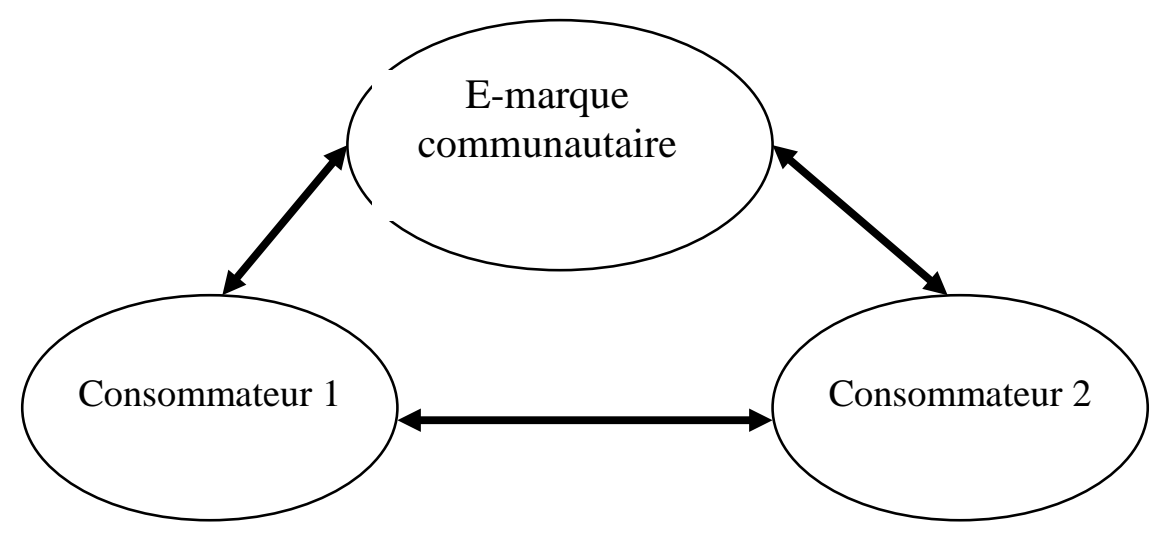

Modèle de la triade relationnelle entre marque communautaire et consommateur

Figure 1 : Relation marque-consommateur (adapté de McAlaxender et al., 2002) 